Supporting Information

\title{
Efficient Synthesis of a Series of Novel Octahydroquinazoline-5-Ones via a Simple on-Water Urea-Catalyzed Chemoselective Five-Component Reaction
}

Sichao Zheng, Shufang Zhong, Zhipeng Chen, Wenhua Chen* and Qiuhua Zhu* Guangdong Provincial Key Laboratory of New Drug Screening, School of Pharmaceutical Sciences, Southern Medical University, 1838 Guangzhou Avenue North, Guangzhou 510515, China.

\section{Contents}

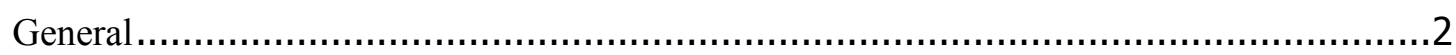

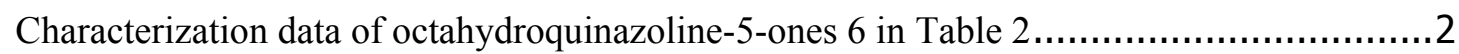

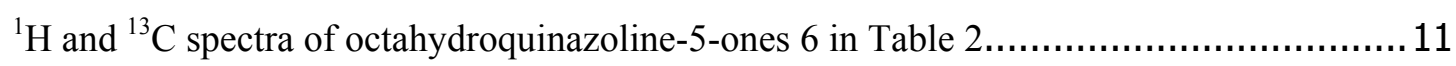




\section{General}

All melting points were taken on a XT-4 melting point apparatus and are uncorrected. ${ }^{1} \mathrm{H}$ NMR (400 MHz) and ${ }^{13} \mathrm{C}$ NMR (100.6 MHz) spectra were recorded using a Bruker Avance $400 \mathrm{MHz}$ NMR spectrometer and respectively referenced to 7.24 and $77.0 \mathrm{ppm}$ for chloroform- $d$ with TMS as internal standard. Mass spectra were recorded on an API 4000QTRAP or on a MAT 95XP. IR spectra were obtained as potassium bromide pellets or as liquid films on potassium bromide pellets with a Bruker Vector 22 spectrometer. Elemental analysis was performed by using an elemental analyzer Vario EL cube. TLC was performed using commercially prepared 100-300 mesh silica gel plates (GF254), and visualization was detected at 254 and $365 \mathrm{~nm}$. All the other chemicals were purchased from Aldrich Chemicals and used without further purification.

\section{Characterization data of octahydroquinazoline-5-ones 6 in Table 2}

\section{1,2,3,4,7,8-hexahydro-2-(4-hydroxyphenyl)-7,7-dimethyl-1,3-}

diphenylquinazolin-5(6H)-one $(\mathbf{6}\{1,1,2\}) 68 \%$ yield, white solid, Mp: $179-180^{\circ} \mathrm{C}$; IR $v_{\max }(\mathrm{KBr}): 3496,2957,2927,1595,1546,1510,1492,1452,1419,1405,1309$, $1265,1167,1104,1074,1036,1007,835,742,700 \mathrm{~cm}^{-1} ;{ }^{1} \mathrm{H}$ NMR (400 MHz, $\mathrm{CDCl}_{3}$ ) $\delta=8.05(\mathrm{~s}, 1 \mathrm{H}), 7.35-7.22(\mathrm{~m}, 7 \mathrm{H}), 7.04(\mathrm{t}, J=6.9 \mathrm{~Hz}, 2 \mathrm{H}), 6.99-6.84(\mathrm{~m}, 5 \mathrm{H})$, $6.11(\mathrm{~s}, 1 \mathrm{H}), 4.44(\mathrm{~d}, J=16.8 \mathrm{~Hz}, 1 \mathrm{H}), 3.69(\mathrm{~d}, J=16.8 \mathrm{~Hz}, 1 \mathrm{H}), 2.37-2.27(\mathrm{~m}, 3 \mathrm{H})$, $2.16(\mathrm{~d}, J=16.8 \mathrm{~Hz}, 1 \mathrm{H}), 1.04(\mathrm{~d}, J=11.3 \mathrm{~Hz}, 6 \mathrm{H}) \mathrm{ppm} ;{ }^{13} \mathrm{C} \mathrm{NMR}\left(101 \mathrm{MHz}, \mathrm{CDCl}_{3}\right)$ $\delta=194.44,157.91,157.09,149.16,143.18,129.49,129.23,129.04,128.13,127.26$ $126.86,121.07,118.68,115.71,104.94,81.72,49.72,41.59,39.53,32.99,28.59$, 28.08 ppm; MS (ESI) $m / z 425\left(M+\mathrm{H}^{+}, 100\right)$ ppm; Anal. Calcd for $\mathrm{C}_{28} \mathrm{H}_{28} \mathrm{~N}_{2} \mathrm{O}_{2}$ : C, 79.22; H, 6.65; N, 6.60. Found: C, 79.10; H, 6.74; N, 6.28.

\section{1,2,3,4,7,8-hexahydro-2-(4-methoxyphenyl)-7,7-dimethyl-1,3-}

diphenylquinazolin-5(6H)-one $(6\{1,1,3\}) 71 \%$ yield, white solid, $\mathrm{Mp}: 169-170^{\circ} \mathrm{C}$; 
IR $v_{\max }(\mathrm{KBr}): 3062,2955,2929,2868,1587,1510,1493,1388,1305,1252,1172$, $1122,1075,1032,1008,930,879,827,755,699 \mathrm{~cm}^{-1} ;{ }^{1} \mathrm{H}$ NMR $\left(400 \mathrm{MHz}, \mathrm{CDCl}_{3}\right) \delta$ $=7.40(\mathrm{~d}, J=8.6 \mathrm{~Hz}, 2 \mathrm{H}), 7.28(\mathrm{~m}, 5 \mathrm{H}), 7.04(\mathrm{~d}, J=8.2 \mathrm{~Hz}, 2 \mathrm{H}), 7.00-6.86(\mathrm{~m}, 5 \mathrm{H})$, $6.16(\mathrm{~s}, 1 \mathrm{H}), 4.40(\mathrm{~d}, J=16.9 \mathrm{~Hz}, 1 \mathrm{H}), 3.85(\mathrm{~s}, 3 \mathrm{H}), 3.64(\mathrm{~d}, J=16.9 \mathrm{~Hz}, 1 \mathrm{H}), 2.37$ (d, $J=16.5 \mathrm{~Hz}, 1 \mathrm{H}), 2.28(\mathrm{~s}, 2 \mathrm{H}), 2.15(\mathrm{~d}, J=16.5 \mathrm{~Hz}, 1 \mathrm{H}), 1.05(\mathrm{~d}, J=10.1 \mathrm{~Hz}, 6 \mathrm{H})$ ppm; ${ }^{13} \mathrm{C}$ NMR $\left(101 \mathrm{MHz}, \mathrm{CDCl}_{3}\right) \delta=193.89,159.56,155.76,149.37,143.73$, $130.72,129.40,129.19,128.14,126.78,126.57,120.87,118.57,114.06,105.96$, $81.10,55.31,50.13,41.65,40.01,33.00,28.83,28.01 \mathrm{ppm}$; MS (ESI) $\mathrm{m} / z 439(M+$ $\mathrm{H}^{+}, 100$ ); Anal. Calcd for $\mathrm{C}_{29} \mathrm{H}_{30} \mathrm{~N}_{2} \mathrm{O}_{2}$ : C, 79.42; H, 6.89; N, 6.39. Found: C, 79.32; H, $6.91 ; \mathrm{N}, 6.26$.

\section{1,2,3,4,7,8-hexahydro-2-(3-hydroxy-4-methoxyphenyl)-7,7-dimethyl-1,3-}

diphenylquinazolin-5(6H)-one $(6\{1,1,4\}) 83 \%$ yield, white solid, Mp: $200-201^{\circ} \mathrm{C}$; IR $v_{\max }(\mathrm{KBr}): 3444,2955,2924,1630,1554,1509,1492,1452,1416,1401,1261$, $1155,1124,1075,1030,931,754,699 \mathrm{~cm}^{-1} ;{ }^{1} \mathrm{H}$ NMR $\left(400 \mathrm{MHz}, \mathrm{CDCl}_{3}\right) \delta=7.34-$ $7.20(\mathrm{~m}, 5 \mathrm{H}), 7.11-6.85(\mathrm{~m}, 8 \mathrm{H}), 6.11(\mathrm{~s}, 1 \mathrm{H}), 5.80(\mathrm{~s}, 1 \mathrm{H}), 4.39(\mathrm{~d}, J=16.9 \mathrm{~Hz}$, 1H), $3.93(\mathrm{~s}, 3 \mathrm{H}), 3.67(\mathrm{~d}, J=16.9 \mathrm{~Hz}, 1 \mathrm{H}), 2.34(\mathrm{~d}, J=16.5 \mathrm{~Hz}, 1 \mathrm{H}), 2.27(\mathrm{~s}, 2 \mathrm{H})$, $2.15(\mathrm{~d}, J=16.5 \mathrm{~Hz}, 1 \mathrm{H}), 1.04(\mathrm{~d}, J=13.3 \mathrm{~Hz}, 6 \mathrm{H}) \mathrm{ppm} ;{ }^{13} \mathrm{C} \mathrm{NMR}\left(101 \mathrm{MHz}, \mathrm{CDCl}_{3}\right)$ $\delta=193.97,155.84,149.37,146.50,145.87,143.73,131.96,129.36,129.17,126.74$ $126.60,120.84,118.55,113.33,110.62,106.04,81.11,56.00,50.10,41.61,40.02$, 32.98, 28.74, 28.06 ppm; MS (ESI) $m / z 455\left(M+\mathrm{H}^{+}, 100\right)$; Anal. Calcd for $\mathrm{C}_{29} \mathrm{H}_{30} \mathrm{~N}_{2} \mathrm{O}_{3}:$ C, 76.63; H, 6.65; N, 6.16. Found: C, 76.60; H, 6.64; N, 5.94.

\section{1,2,3,4,7,8-hexahydro-2-(4-hydroxy-3,5-dimethoxyphenyl)-7,7-dimethyl-1,3-}

diphenylquinazolin-5(6H)-one $(\mathbf{6}\{\mathbf{1 , 1 , 5}\}) 61 \%$ yield, white solid, Mp: $194-195^{\circ} \mathrm{C}$; IR $v_{\max }(\mathrm{KBr}): \quad 3413,3060,2955,2931,1599,1586,1567,1516,1493,1454,1404$, 1366, 1319, 1259, 1213, 1178, 1146, 1115, 1076, 1032, 1009, 918, 836, 764, 733, 699 
$\mathrm{cm}^{-1} ;{ }^{1} \mathrm{H}$ NMR $\left(400 \mathrm{MHz}, \mathrm{CDCl}_{3}\right) \delta=7.32(\mathrm{~m}, 2 \mathrm{H}), 7.28-7.21(\mathrm{~m}, 3 \mathrm{H}), 7.09-6.88$ (m, 5H), $6.73(\mathrm{~s}, 2 \mathrm{H}), 6.13(\mathrm{~s}, 1 \mathrm{H}), 5.62(\mathrm{~s}, 1 \mathrm{H}), 4.42(\mathrm{~d}, J=16.9 \mathrm{~Hz}, 1 \mathrm{H}), 3.87(\mathrm{~s}$, $6 \mathrm{H}), 3.71(\mathrm{~d}, J=16.9 \mathrm{~Hz}, 1 \mathrm{H}), 2.44(\mathrm{~d}, J=16.4 \mathrm{~Hz}, 1 \mathrm{H}), 2.32(\mathrm{~d}, J=16.5 \mathrm{~Hz}, 1 \mathrm{H})$, $2.24(\mathrm{~d}, J=16.5 \mathrm{~Hz}, 1 \mathrm{H}), 2.09(\mathrm{~d}, J=16.4 \mathrm{~Hz}, 1 \mathrm{H}), 1.06(\mathrm{~d}, J=16.0 \mathrm{~Hz}, 6 \mathrm{H}) ;{ }^{13} \mathrm{C}$ NMR (101 MHz, $\left.\mathrm{CDCl}_{3}\right) \delta=193.82,155.48,149.21,147.20,143.90,134.77,129.97$, $129.46,129.21,126.82,126.37,121.00,118.59,106.26,103.80,81.40,56.36,50.07$, 41.89, 40.11, 33.04, 29.31, 27.58 ppm; MS (ESI) $m / z 485\left(M+\mathrm{H}^{+}, 100\right)$; Anal. Calcd for $\mathrm{C}_{30} \mathrm{H}_{32} \mathrm{~N}_{2} \mathrm{O}_{4}$ : C, 74.36; H, 6.66; N, 5.78. Found: C, 74.19; H, 6.66; N, 5.54.

\section{1,2,3,4,7,8-hexahydro-7,7-dimethyl-1,3-diphenyl-2-(thiophen-2-yl)quinazolin-}

5(6H)-one $(\mathbf{6}\{\mathbf{1 , 1 , 6}\}) 80 \%$ yield, argenteous solid; Mp: $164-165^{\circ} \mathrm{C}$; IR $v_{\max }(\mathrm{KBr})$ : 2953, 2925, 2867, 1626, 1574, 1492, 1452, 1400, 1386, 1315, 1291, 1257, 1174, 1148 , $1121,1075,1031,1008,929,754,698 \mathrm{~cm}^{-1} ;{ }^{1} \mathrm{H}$ NMR $\left(400 \mathrm{MHz}, \mathrm{CDCl}_{3}\right) \delta=7.31(\mathrm{~m}$, 6H), $7.11-7.02(\mathrm{~m}, 4 \mathrm{H}), 7.02-6.97(\mathrm{~m}, 2 \mathrm{H}), 6.94(\mathrm{t}, J=7.3 \mathrm{~Hz}, 1 \mathrm{H}), 6.35(\mathrm{~s}, 1 \mathrm{H})$, $4.40(\mathrm{~d}, J=16.7 \mathrm{~Hz}, 1 \mathrm{H}), 3.88$ (d, $J=16.7 \mathrm{~Hz}, 1 \mathrm{H}), 2.34$ (d, $J=16.5 \mathrm{~Hz}, 1 \mathrm{H}), 2.29$ (s, 2H), $2.11(\mathrm{~d}, J=16.5 \mathrm{~Hz}, 1 \mathrm{H}), 1.05(\mathrm{~d}, J=5.1 \mathrm{~Hz}, 6 \mathrm{H}) \mathrm{ppm} ;{ }^{13} \mathrm{C}$ NMR $(101 \mathrm{MHz}$, $\left.\mathrm{CDCl}_{3}\right) \delta=194.27,155.11,148.44,143.63,142.99,129.49,129.25,127.02,126.90$ $126.68,125.86,125.67,121.14,118.37,107.05,78.01,50.23,41.64,40.47,32.93$, 28.64, 28.15 ppm; MS (ESI) $m / z 415\left(M+\mathrm{H}^{+}, 100\right)$; Anal. Calcd for $\mathrm{C}_{26} \mathrm{H}_{26} \mathrm{~N}_{2} \mathrm{OS}$ : C, 75.33; H, 6.32; N, 6.76. Found: C, 75.20; H, 6.54; N, 6.52.

\section{2-(5-bromothiophen-2-yl)-1,2,3,4,7,8-hexahydro-7,7-dimethyl-1,3-}

diphenylquinazolin-5(6H)-one $(\mathbf{6}\{1,1,7\}) 83 \%$ yield, yellow solid, Mp: $76-77^{\circ} \mathrm{C}$; IR $v_{\max }(\mathrm{KBr}): 2955,2925,1637,1577,1491,1458,1399,1310,1257,1117,1202,1174$, $1157,1122,1072,1009,932,882,824,737,668 \mathrm{~cm}^{-1} ;{ }^{1} \mathrm{H}$ NMR (400 MHz, $\left.\mathrm{CDCl}_{3}\right) \delta$ $=7.39-7.24(\mathrm{~m}, 5 \mathrm{H}), 6.98(\mathrm{~m}, 6 \mathrm{H}), 6.79(\mathrm{~m}, 1 \mathrm{H}), 6.23(\mathrm{~s}, 1 \mathrm{H}), 4.38(\mathrm{~d}, J=16.9 \mathrm{~Hz}$, 1H), $3.90(\mathrm{~d}, J=16.9 \mathrm{~Hz}, 1 \mathrm{H}), 2.36-2.28(\mathrm{~m}, 3 \mathrm{H}), 2.09(\mathrm{~d}, J=16.6 \mathrm{~Hz}, 1 \mathrm{H}), 1.04(\mathrm{~d}$, 
$J=2.8 \mathrm{~Hz}, 6 \mathrm{H}) \mathrm{ppm} ;{ }^{13} \mathrm{C} \mathrm{NMR}\left(101 \mathrm{MHz}, \mathrm{CDCl}_{3}\right) \delta=194.39,154.84,148.16$, $144.40,143.53,129.76,129.59,129.32,129.14,127.16,126.59,125.91,121.34$, $120.91,118.28,112.49,107.54,77.77,53.37,50.22,41.56,40.56,32.88,28.43,28.27$ ppm; MS (ESI) $m / z 493\left(M+\mathrm{H}^{+}, 100\right)$; Anal. Calcd for $\mathrm{C}_{26} \mathrm{H}_{25} \mathrm{BrN}_{2} \mathrm{OS}$ : C, 63.28; $\mathrm{H}$, 5.11; N, 5.68. Found: C, 62.78; H, 5.13; N, 5.46.

2-(4-bromophenyl)-1,2,3,4,7,8-hexahydro-7,7-dimethyl-1,3-diphenylquinazolin$\mathbf{5 ( 6 H )}$-one $(\mathbf{6}\{\mathbf{1 , 1 , 8}\}) 73 \%$ yield, white solid, Mp: 197-198 ${ }^{\circ} \mathrm{C}$; IR $v_{\max }(\mathrm{KBr}): 3058$, 2954, 2925, 1626, 1569, 1491, 1454, 1390, 1302, 1257, 1173, 1150, 1121, 1072, 1034, 1009, 933, 759, $697 \mathrm{~cm}^{-1} ;{ }^{1} \mathrm{H}$ NMR $\left(400 \mathrm{MHz}, \mathrm{CDCl}_{3}\right) \delta=7.56(\mathrm{~d}, J=8.4 \mathrm{~Hz}, 2 \mathrm{H})$, $7.37(\mathrm{~d}, J=8.4 \mathrm{~Hz}, 2 \mathrm{H}), 7.34-7.21(\mathrm{~m}, 5 \mathrm{H}), 7.02(\mathrm{~d}, \mathrm{~J}=8.1 \mathrm{~Hz}, 2 \mathrm{H}), 6.93(\mathrm{~m}, 3 \mathrm{H})$, $6.13(\mathrm{~s}, 1 \mathrm{H}), 4.40(\mathrm{~d}, J=17.1 \mathrm{~Hz}, 1 \mathrm{H}), 3.59(\mathrm{~d}, J=17.1 \mathrm{~Hz}, 1 \mathrm{H}), 2.38(\mathrm{~d}, J=16.5 \mathrm{~Hz}$, 1H), $2.28(\mathrm{~s}, 2 \mathrm{H}), 2.15(\mathrm{~d}, J=16.5 \mathrm{~Hz}, 1 \mathrm{H}), 1.04(\mathrm{~d}, J=4.5 \mathrm{~Hz}, 6 \mathrm{H}) ;{ }^{13} \mathrm{C}$ NMR $(101$ $\left.\mathrm{MHz}, \mathrm{CDCl}_{3}\right) \delta=193.99,155.53,149.15,143.58,137.88,131.91,129.54,129.28$, $128.72,126.95,126.45,122.38,121.20,118.57,106.35,80.95,50.11,41.63,40.20$, 33.04, 28.78, $28.02 \mathrm{ppm}$; MS (ESI) $m / z 487\left(M+\mathrm{H}^{+}, 100\right)$; Anal. Calcd for $\mathrm{C}_{28} \mathrm{H}_{27} \mathrm{BrN}_{2} \mathrm{O}$ : C, 68.99; H, 5.58; N, 5.75. Found: C, 69.01; H, 5.64; N, 5.58.

\section{2-(4-(trifluoromethyl)phenyl)-1,2,3,4,7,8-hexahydro-7,7-dimethyl-1,3-}

diphenylquinazolin-5(6H)-one $(\mathbf{6}\{\mathbf{1 , 1 , 9}\}) 84 \%$ yield, white solid; MP: $192-193^{\circ} \mathrm{C}$; IR $v_{\max }(\mathrm{KBr}): 3065,2958,2931,2865,1580,1504,1496,1388,1302,1249,1176$, 1122, 1074, 1033, 1005, 930, 877, 824, 765, $699 \mathrm{~cm}^{-1} ;{ }^{1} \mathrm{H}$ NMR (400 MHz, $\left.\mathrm{CDCl}_{3}\right) \delta$ $=7.70(\mathrm{~d}, J=8.3 \mathrm{~Hz}, 2 \mathrm{H}), 7.63(\mathrm{~d}, J=8.3 \mathrm{~Hz}, 2 \mathrm{H}), 7.36-7.23(\mathrm{~m}, 5 \mathrm{H}), 7.04(\mathrm{~d}, J=$ $8.1 \mathrm{~Hz}, 2 \mathrm{H}), 6.97(\mathrm{t}, J=7.3 \mathrm{~Hz}, 1 \mathrm{H}), 6.91(\mathrm{~d}, J=7.3 \mathrm{~Hz}, 2 \mathrm{H}), 6.21(\mathrm{~s}, 1 \mathrm{H}), 4.41$ (d, $J$ $=17.2 \mathrm{~Hz}, 1 \mathrm{H}), 3.55(\mathrm{~d}, J=17.2 \mathrm{~Hz}, 1 \mathrm{H}), 2.40(\mathrm{~d}, J=16.5 \mathrm{~Hz}, 1 \mathrm{H}), 2.29(\mathrm{~s}, 2 \mathrm{H})$, $2.17(\mathrm{~d}, J=16.5 \mathrm{~Hz}, 1 \mathrm{H}), 1.06(\mathrm{~d}, J=4.8 \mathrm{~Hz}, 6 \mathrm{H}) \mathrm{ppm} ;{ }^{13} \mathrm{C} \mathrm{NMR}\left(101 \mathrm{MHz}, \mathrm{CDCl}_{3}\right)$ $\delta=194.09,155.51,149.09,143.58,129.85,129.58,129.32,129.19,129.04,128.90$ 
$127.38,127.02,126.44,125.81,124.39,121.33,120.82,118.57,117.60,106.56$ 99.93, 81.01, 50.08, 41.63, 40.30, 33.02, 32.63, 28.72, 28.25, 28.02 ppm; MS (ESI) $m / z 477\left(M+\mathrm{H}^{+}, 100\right)$; Anal. Calcd for $\mathrm{C}_{29} \mathrm{H}_{27} \mathrm{~F}_{3} \mathrm{~N}_{2} \mathrm{O}$ : C, 73.09; H, 5.71; N, 5.88; Found: C, 73.43; H, 5.90; N, 5.64.

\section{4-(1,2,3,4,5,6,7,8-octahydro-7,7-dimethyl-5-oxo-1,3-diphenylquinazolin-2-}

yl)benzonitrile $(\mathbf{6}\{\mathbf{1 , 1 , 1 0}\}) 74 \%$ yield, white solid, Mp: $184-185^{\circ} \mathrm{C}$; IR $v_{\max }(\mathrm{KBr})$ : 2956, 2926, 2869, 2228, 1626, 1575, 1493, 1453, 1388, 1308, 1257, 1194, 1174, 1155, 1123, 1075, 1033, 1015, 932, 884, 827, 766, 747, $698 \mathrm{~cm}^{-1} ;{ }^{1} \mathrm{H}$ NMR (400 MHz, $\left.\mathrm{CDCl}_{3}\right) \delta=7.78-7.68(\mathrm{~m}, 2 \mathrm{H}), 7.60(\mathrm{~d}, J=8.1 \mathrm{~Hz}, 2 \mathrm{H}), 7.28(\mathrm{~m}, 5 \mathrm{H}), 7.05-6.80$ (m, 5H), $6.16(\mathrm{~s}, 1 \mathrm{H}), 4.37(\mathrm{~d}, J=17.3 \mathrm{~Hz}, 1 \mathrm{H}), 3.49$ (d, $J=17.3 \mathrm{~Hz}, 1 \mathrm{H}), 2.35(\mathrm{~d}, J$ $=16.5 \mathrm{~Hz}, 1 \mathrm{H}), 2.26(\mathrm{~s}, 2 \mathrm{H}), 2.14(\mathrm{~d}, J=16.5 \mathrm{~Hz}, 1 \mathrm{H}), 1.02(\mathrm{~d}, J=1.2 \mathrm{~Hz}, 6 \mathrm{H}) \mathrm{ppm}$;

${ }^{13} \mathrm{C}$ NMR $\left(101 \mathrm{MHz}, \mathrm{CDCl}_{3}\right) \delta=194.11,155.45,148.93,144.13,143.44,132.63$, $129.66,129.38,127.80,127.16,126.44,121.52,118.57,118.36,112.39,106.66$, 81.02, 50.08, 41.58, 40.32, 33.01, 28.59, $28.12 \mathrm{ppm}$; MS (ESI) $m / z 456\left(M+\mathrm{Na}^{+}\right.$, 100); Anal. Calcd for $\mathrm{C}_{29} \mathrm{H}_{27} \mathrm{~N}_{3} \mathrm{O}$ : C, 80.34; H, 6.28; N, 9.69. Found: C, 79.94; H, $6.28 ; \mathrm{N}, 9.61$.

\section{1,2,3,4,7,8-hexahydro-7,7-dimethyl-2-(4-nitrophenyl)-1,3-diphenylquinazolin-}

5(6H)-one $(6\{1,1,11\}) 65 \%$ yield, yellow solid, Mp: 182-183 ${ }^{\circ} \mathrm{C}$; IR $v_{\max }(\mathrm{KBr}): 2956$, 2926, 2868, 1627, 1573, 1521, 1492, 1452, 1414, 1387, 1367, 1346, 1294, 1256, 1188, $1175,1157,1123,1112,1075,1032,1011,912,830,761,740,698 \mathrm{~cm}^{-1} ;{ }^{1} \mathrm{H}$ NMR $\left(400 \mathrm{MHz}, \mathrm{CDCl}_{3}\right) \delta=8.35-8.25(\mathrm{~m}, 2 \mathrm{H}), 7.69(\mathrm{~d}, J=8.4 \mathrm{~Hz}, 2 \mathrm{H}), 7.36-7.26(\mathrm{~m}$, 5H), $7.07-6.86(\mathrm{~m}, 5 \mathrm{H}), 6.23(\mathrm{~s}, 1 \mathrm{H}), 4.41(\mathrm{~d}, J=17.3 \mathrm{~Hz}, 1 \mathrm{H}), 3.52(\mathrm{~d}, J=17.3 \mathrm{~Hz}$, 1H), $2.39(\mathrm{~d}, J=16.6 \mathrm{~Hz}, 1 \mathrm{H}), 2.29(\mathrm{~s}, 2 \mathrm{H}), 2.18(\mathrm{~d}, J=16.6 \mathrm{~Hz}, 1 \mathrm{H}), 1.06(\mathrm{~d}, J=4.5$ $\mathrm{Hz}, 6 \mathrm{H}) \mathrm{ppm} ;{ }^{13} \mathrm{C} \mathrm{NMR}\left(101 \mathrm{MHz}, \mathrm{CDCl}_{3}\right) \delta=194.15,155.48,148.87,147.94$, $145.98,143.39,129.70,129.41,128.02,127.23,126.46,124.05,121.61,118.59$, 
$106.68,80.91,50.06,41.59,40.35,33.02,28.59,28.13$ ppm; MS (ESI) $m / z 476(M+$ $\mathrm{Na}^{+}, 100$ ); Anal. Calcd for $\mathrm{C}_{28} \mathrm{H}_{27} \mathrm{~N}_{3} \mathrm{O}_{3}$ : C, 74.15; H, 6.00; N, 9.27. Found: C, 73.82; H, 5.93; N, 9.26.

\section{2-butyl-1,2,3,4,7,8-hexahydro-7,7-dimethyl-1,3-diphenylquinazolin-5(6H)-one}

(6\{1,1,12\}) $85 \%$ yield, white solid, Mp: 110-111 ${ }^{\circ} \mathrm{C}$; IR $v_{\max }(\mathrm{KBr}): 3060,2955,2869$, $1621,1568,1493,1452,1423,1395,1317,1290,1263,1199,1175,1150,1073,1007$, 995, 760, 699, $668 \mathrm{~cm}^{-1} ;{ }^{1} \mathrm{H}$ NMR (400 MHz, $\left.\mathrm{CDCl}_{3}\right) \delta=7.42-7.22(\mathrm{~m}, 5 \mathrm{H}), 7.08-$ $6.75(\mathrm{~m}, 5 \mathrm{H}), 5.02(\mathrm{~m}, 1 \mathrm{H}), 4.50(\mathrm{~d}, J=17.1 \mathrm{~Hz}, 1 \mathrm{H}), 4.05(\mathrm{~d}, J=17.1 \mathrm{~Hz}, 1 \mathrm{H}), 2.32$ $(\mathrm{d}, J=16.4 \mathrm{~Hz}, 1 \mathrm{H}), 2.23(\mathrm{~d}, J=16.4 \mathrm{~Hz}, 1 \mathrm{H}), 2.14-2.00(\mathrm{~m}, 2 \mathrm{H}), 1.91-1.75(\mathrm{~m}$, 2H), $1.62-1.46(\mathrm{~m}, 1 \mathrm{H}), 1.45-1.25(\mathrm{~m}, 3 \mathrm{H}), 0.93(\mathrm{~m}, 9 \mathrm{H}) \mathrm{ppm} ;{ }^{13} \mathrm{C}$ NMR $(101$ $\left.\mathrm{MHz}, \mathrm{CDCl}_{3}\right) \delta=193.56,155.93,150.16,142.87,129.53,129.13,127.92,127.31$, $120.71,118.61,103.13,80.73,49.97,41.42,38.70,32.71,31.04,29.27,27.32,26.93$, 22.32, 13.97 ppm; MS (ESI) $m / z 389\left(M+\mathrm{H}^{+}, 100\right)$; Anal. Calcd for $\mathrm{C}_{26} \mathrm{H}_{32} \mathrm{~N}_{2} \mathrm{O}: \mathrm{C}$, 80.37; H, 8.30; N, 7.21. Found: C, 80.15; H, 8.33; N, 7.10.

\section{1,2,3,4,7,8-hexahydro-2-isopropyl-7,7-dimethyl-1,3-diphenylquinazolin-5(6H)-}

one $(6\{1,1,13\}) 83 \%$ yield, white solid; Mp: $135-136^{\circ} \mathrm{C}$; IR $v_{\max }(\mathrm{KBr}): 2958,2869$, 2360, 2342, 1591, 1574, 1493, 1469, 1453, 1397, 1308, 1290, 1251, 1172, 1150, 1061, 1033, 1008, 993, 963, 916, 841, 760, 749, 699, $669 \mathrm{~cm}^{-1} ;{ }^{1} \mathrm{H}$ NMR (400 MHz, $\left.\mathrm{CDCl}_{3}\right)$ $\delta=7.26(\mathrm{~m}, 2 \mathrm{H}), 7.18(\mathrm{~m}, 3 \mathrm{H}), 6.82(\mathrm{~m}, 5 \mathrm{H}), 4.66(\mathrm{~d}, J=9.8 \mathrm{~Hz}, 1 \mathrm{H}), 4.36(\mathrm{~d}, J=$ $17.1 \mathrm{~Hz}, 1 \mathrm{H}), 4.04(\mathrm{~d}, J=17.1 \mathrm{~Hz}, 1 \mathrm{H}), 2.47-2.20(\mathrm{~m}, 3 \mathrm{H}), 2.08(\mathrm{~m}, 2 \mathrm{H}), 1.13(\mathrm{~d}, J$ $=6.7 \mathrm{~Hz}, 3 \mathrm{H}), 0.98(\mathrm{~m}, 9 \mathrm{H}) \mathrm{ppm} ;{ }^{13} \mathrm{C} \mathrm{NMR}\left(101 \mathrm{MHz}, \mathrm{CDCl}_{3}\right) \delta=194.60,156.20$, $149.91,145.18,129.20,129.12,127.19,126.34,119.82,117.55,107.97,85.94,50.30$, 41.97, 39.89, 32.78, 30.75, 28.85, 27.59, 20.73, 19.16 ppm; MS (ESI) $\mathrm{m} / z 375(M+$ $\mathrm{H}^{+}, 100$ ); Anal. Calcd for $\mathrm{C}_{25} \mathrm{H}_{30} \mathrm{~N}_{2} \mathrm{O}: \mathrm{C}, 80.17 ; \mathrm{H}, 8.07$; N, 7.48; Found: C, 80.45; $\mathrm{H}$, $8.06 ; \mathrm{N}, 7.23$. 
3-(4-bromophenyl)-1,2,3,4,7,8-hexahydro-7,7-dimethyl-1,2-diphenylquinazolin5(6H)-one $(\mathbf{6}\{\mathbf{1 , 2 , 1}\}) 82 \%$ yield, white solid; Mp: $169-170^{\circ} \mathrm{C}$; IR $v_{\max }(\mathrm{KBr}): 2954$, 2924, 2853, 1626, 1587, 1572, 1491, 1462, 1453, 1387, 1308, 1256, 1175, 1121, 1089, 1009, 820, 742, $699 \mathrm{~cm}^{-1} ;{ }^{1} \mathrm{H}$ NMR (400 MHz, $\left.\mathrm{CDCl}_{3}\right) \delta=7.50-7.24(\mathrm{~m}, 10 \mathrm{H}), 7.03$ $-6.86(\mathrm{~m}, 4 \mathrm{H}), 6.14(\mathrm{~d}, J=1.2 \mathrm{~Hz}, 1 \mathrm{H}), 4.34(\mathrm{~d}, J=17.0 \mathrm{~Hz}, 1 \mathrm{H}), 3.60(\mathrm{~d}, J=17.0$ $\mathrm{Hz}, 1 \mathrm{H}), 2.38(\mathrm{~d}, J=16.5 \mathrm{~Hz}, 1 \mathrm{H}), 2.28(\mathrm{~s}, 2 \mathrm{H}), 2.17(\mathrm{~d}, J=16.5 \mathrm{~Hz}, 1 \mathrm{H}), 1.05(\mathrm{~d}, J$ $=9.2 \mathrm{~Hz}, 6 \mathrm{H}) \mathrm{ppm} ;{ }^{13} \mathrm{C} \mathrm{NMR}\left(101 \mathrm{MHz}, \mathrm{CDCl}_{3}\right) \delta=193.99,155.77,148.47,143.54$, $138.33,132.07,129.56,128.84,128.80,128.50,126.99,126.83,126.50,120.14$ 113.09, 105.76, 81.16, 50.07, 41.61, 40.13, 33.02, 28.82, 27.97 ppm; MS (ESI) m/z $487\left(M+\mathrm{H}^{+}, 100\right)$; Anal. Calcd for $\mathrm{C}_{28} \mathrm{H}_{27} \mathrm{BrN}_{2} \mathrm{O}: \mathrm{C}, 68.99 ; \mathrm{H}, 5.58$; N, 5.75; Found: C, 69.40; H, 5.72; N, 5.37.

\section{1,2,3-tris(4-bromophenyl)-1,2,3,4,7,8-hexahydro-7,7-dimethylquinazolin-5(6H)-} one $(6\{2,2,8\}) 66 \%$ yield, white solid, Mp: $178-179^{\circ} \mathrm{C}$; IR $v_{\max }(\mathrm{KBr}): 2956,2927$, $2868,1627,1579,1566,1488,1417,1387,1367,1304,1256,1202,1174,1157,1122$, $1072,1009,932,882,824,737,668 \mathrm{~cm}^{-1} ;{ }^{1} \mathrm{H}$ NMR $\left(400 \mathrm{MHz}, \mathrm{CDCl}_{3}\right) \delta=7.56(\mathrm{ppm})$ $(\mathrm{d}, J=8.5 \mathrm{~Hz}, 2 \mathrm{H}), 7.45(\mathrm{~d}, J=8.7 \mathrm{~Hz}, 2 \mathrm{H}), 7.39-7.29(\mathrm{~m}, 4 \mathrm{H}), 6.86(\mathrm{~d}, J=8.9 \mathrm{~Hz}$, 2H), $6.78(\mathrm{~d}, J=8.6 \mathrm{~Hz}, 2 \mathrm{H}), 6.02(\mathrm{~s}, 1 \mathrm{H}), 4.30(\mathrm{~d}, J=17.2 \mathrm{~Hz}, 1 \mathrm{H}), 3.54(\mathrm{~d}, J=17.2$ $\mathrm{Hz}, 1 \mathrm{H}), 2.38(\mathrm{~d}, J=16.5 \mathrm{~Hz}, 1 \mathrm{H}), 2.27(\mathrm{~d}, J=1.5 \mathrm{~Hz}, 2 \mathrm{H}), 2.11(\mathrm{~d}, J=16.5 \mathrm{~Hz}, 1 \mathrm{H})$, 1.04 (s, 6H) ppm; ${ }^{13} \mathrm{C}$ NMR (101 MHz, $\left.\mathrm{CDCl}_{3}\right) \delta=194.13,154.72,148.10,142.53$, $137.17,132.81,132.21,132.11,128.49,127.77,122.75,120.59,120.14,113.58$ 106.86, 80.77, 50.04, 41.63, 40.22, 33.12, 28.85, 27.87 ppm; MS (ESI) $m / z 667$ (M + $\mathrm{Na}^{+}$, 100); Anal. Calcd for $\mathrm{C}_{28} \mathrm{H}_{25} \mathrm{Br}_{3} \mathrm{~N}_{2} \mathrm{O}: \mathrm{C}, 52.12 ; \mathrm{H}, 3.91 ; \mathrm{N}, 4.34$. Found: C, $52.04 ; \mathrm{H}, 3.95 ; \mathrm{N}, 4.16$.

\section{1,2,3,4,7,8-hexahydro-3-(4-methoxyphenyl)-7,7-dimethyl-1,2-}

diphenylquinazolin-5(6H)-one $(\mathbf{6}\{\mathbf{1 , 3 , 1}\}) 80 \%$ yield, white solid, Mp:134-135 ${ }^{\circ}$; IR 
$v_{\max }(\mathrm{KBr}): 2956,1684,1653,1624,1570,1560,1541,1509,1491,1457,1473,1437$, $1388,1294,1257,1176,1092,1036,933,826,757,723,700,648 \mathrm{~cm}^{-1} ;{ }^{1} \mathrm{H}$ NMR $\left(400 \mathrm{MHz}, \mathrm{CDCl}_{3}\right) \delta=7.56-7.33(\mathrm{~m}, 5 \mathrm{H}), 7.31-7.20(\mathrm{~m}, 3 \mathrm{H}), 7.07-6.97(\mathrm{~m}, 2 \mathrm{H})$, $6.95-6.74(\mathrm{~m}, 4 \mathrm{H}), 6.02(\mathrm{~s}, 1 \mathrm{H}), 4.27(\mathrm{~d}, J=17.1 \mathrm{~Hz}, 1 \mathrm{H}), 3.80(\mathrm{~s}, 3 \mathrm{H}), 3.59(\mathrm{~d}, J=$ $17.1 \mathrm{~Hz}, 1 \mathrm{H}), 2.44-2.23(\mathrm{~m}, 3 \mathrm{H}), 2.17(\mathrm{~d}, J=16.4 \mathrm{~Hz}, 1 \mathrm{H}), 1.03(\mathrm{t}, J=17.8 \mathrm{~Hz}, 6 \mathrm{H})$ ppm; ${ }^{13} \mathrm{C}$ NMR $\left(101 \mathrm{MHz}, \mathrm{CDCl}_{3}\right) \delta=193.90,155.97,154.37,143.73,143.33$, $138.76,129.37,128.66,128.27,126.98,126.79,126.73,120.63,114.39,105.75$, 82.96, 55.48, 50.12, 41.62, 40.38, 32.94, 28.80, 28.10 ppm; MS (ESI) $m / z 439(M+$ $\left.\mathrm{H}^{+}, 100\right)$; Anal. Calcd for $\mathrm{C}_{29} \mathrm{H}_{30} \mathrm{~N}_{2} \mathrm{O}_{2}: \mathrm{C}, 79.42 ; \mathrm{H}, 6.89 ; \mathrm{N}, 6.39$; Found: C, 79.41; H, $6.98 ; \mathrm{N}, 6.43$.

\section{1,2,3,4,7,8-hexahydro-7,7-dimethyl-1-(naphthalen-1-yl)-2,3-}

diphenylquinazolin-5(6H)-one $(\mathbf{6}\{3,1,1\}) 74 \%$ yield, white solid, Mp: $191-192^{\circ} \mathrm{C}$; IR $v_{\max }(\mathrm{KBr}): 3056,2956,2870,1622,1567,1497,1464,1420,1384,1313,1294$, $1264,1178,1154,1125,1068,1014,985,949,914,805,779,756,698 \mathrm{~cm}^{-1} ;{ }^{1} \mathrm{H}$ $\operatorname{NMR}\left(400 \mathrm{MHz}, \mathrm{CDCl}_{3}\right) \delta=7.89(\mathrm{~d}, J=8.2 \mathrm{~Hz}, 1 \mathrm{H}), 7.84(\mathrm{~d}, J=8.2 \mathrm{~Hz}, 1 \mathrm{H}), 7.50$ $(\mathrm{t}, J=7.5 \mathrm{~Hz}, 1 \mathrm{H}), 7.47-7.23(\mathrm{~m}, 10 \mathrm{H}), 7.21(\mathrm{~d}, J=7.2 \mathrm{~Hz}, 1 \mathrm{H}), 7.09(\mathrm{~d}, J=8.0 \mathrm{~Hz}$, 2H), $6.97(\mathrm{t}, J=7.3 \mathrm{~Hz}, 1 \mathrm{H}), 6.11(\mathrm{~s}, 1 \mathrm{H}), 4.54(\mathrm{~d}, J=16.4 \mathrm{~Hz}, 1 \mathrm{H}), 3.76(\mathrm{~d}, J=16.4$ Hz, 1H), 2.39 (d, $J=16.3 \mathrm{~Hz}, 1 \mathrm{H}), 2.31$ (d, $J=16.3 \mathrm{~Hz}, 1 \mathrm{H}), 2.25$ (d, $J=16.9 \mathrm{~Hz}$, 1H), $1.85(\mathrm{~d}, \mathrm{~J}=16.9 \mathrm{~Hz}, 1 \mathrm{H}), 1.02(\mathrm{~s}, 3 \mathrm{H}), 0.95$ (s, 3H) ppm; ${ }^{13} \mathrm{C}$ NMR (101 MHz, $\left.\mathrm{CDCl}_{3}\right) \delta=193.41,158.00,149.08,138.55,138.15,134.62,130.57,129.36,128.84$, $128.50,128.41,127.45,127.12,126.73,125.21,122.34,121.13,118.79,103.47$ 81.77, 50.02, 40.49, 38.42, 32.37, 29.15, $27.47 \mathrm{ppm}$; MS (ESI) $m / z 459\left(M+\mathrm{H}^{+}, 100\right)$; Anal. Calcd for $\mathrm{C}_{32} \mathrm{H}_{30} \mathrm{~N}_{2} \mathrm{O}$ : C, 83.81; H, 6.59; N, 6.11; Found: C, 83.86; H, 6.69; N, 5.97. 


\section{1,2,3,4,7,8-hexahydro-7,7-dimethyl-1-(diphenylamine-4-yl)-2,3-}

diphenylquinazolin-5(6H)-one $(\mathbf{6}\{4,1,1\}) 70 \%$ yield, yellow solid, Mp: $192-193^{\circ} \mathrm{C}$; IR $v_{\max }(\mathrm{KBr}): 2956,2360,2342,1596,1558,1508,1496,1449,1419,1398,1314$, $1260,1174,1075,1019,933,836,745,696,669 \mathrm{~cm}^{-1} ;{ }^{1} \mathrm{H}$ NMR $\left(400 \mathrm{MHz}, \mathrm{CDCl}_{3}\right) \delta$ $=7.57-7.20(\mathrm{~m}, 9 \mathrm{H}), 7.16-6.84(\mathrm{~m}, 8 \mathrm{H}), 6.78(\mathrm{~d}, J=8.4 \mathrm{~Hz}, 2 \mathrm{H}), 6.17(\mathrm{~s}, 1 \mathrm{H})$, $5.84(\mathrm{~s}, 1 \mathrm{H}), 4.41(\mathrm{~d}, J=16.9 \mathrm{~Hz}, 1 \mathrm{H}), 3.60(\mathrm{~d}, J=16.9 \mathrm{~Hz}, 1 \mathrm{H}), 2.38-2.15(\mathrm{~m}, 4 \mathrm{H})$, $1.06(\mathrm{~d}, J=20.1 \mathrm{~Hz}, 6 \mathrm{H}) \mathrm{ppm} ;{ }^{13} \mathrm{C} \mathrm{NMR}\left(101 \mathrm{MHz}, \mathrm{CDCl}_{3}\right) \delta=193.61,156.77$, $149.45,142.58,141.95,138.73,135.88,129.38,129.21,128.66,128.27,128.02$, $126.96,121.95,120.94,118.81,118.70,117.04,104.95,81.88,50.01,41.46,39.69$, 32.80, 28.66, 28.28 ppm; MS (ESI) $m / z 500\left(M+\mathrm{H}^{+}, 100\right)$; Anal. Calcd for $\mathrm{C}_{34} \mathrm{H}_{33} \mathrm{~N}_{3} \mathrm{O}$ : C, 81.73; H, 6.66; N, 8.41; Found: C, 81.48; H, 6.72; N, 8.15.

\section{3-benzyl-1,2,3,4,7,8-hexahydro-7,7-dimethyl-1,2-diphenylquinazolin-5(6H)-one}

(6 $\{1,4,1\}) 72 \%$ yield, white solid, Mp: $102-103^{\circ} \mathrm{C}$; IR $v_{\max }(\mathrm{KBr}): 3061,2955,1653$, $1617,1587,1570,1559,1540,1533,1522,1491,1456,1472,1437,1418,1388,1364$, 1292, 1273, 1174, 1154, 1100, 1062, 1028, 1010, 741, $699 \mathrm{~cm}^{-1} ;{ }^{1} \mathrm{H}$ NMR (400 MHz, $\left.\mathrm{CDCl}_{3}\right) \delta=7.56-6.93(\mathrm{~m}, 15 \mathrm{H}), 5.19(\mathrm{~s}, 1 \mathrm{H}), 3.91(\mathrm{~s}, 2 \mathrm{H}), 3.64(\mathrm{~d}, J=17.1 \mathrm{~Hz}, 1 \mathrm{H})$, $3.37(\mathrm{~d}, J=17.1 \mathrm{~Hz}, 1 \mathrm{H}), 2.61(\mathrm{~d}, J=16.4 \mathrm{~Hz}, 1 \mathrm{H}), 2.33(\mathrm{~d}, J=16.8 \mathrm{~Hz}, 1 \mathrm{H}), 2.26(\mathrm{~d}$, $J=16.8,1 \mathrm{H}), 2.25(\mathrm{~d}, J=16.4,1 \mathrm{H}), 1.12(\mathrm{~d}, J=24.5 \mathrm{~Hz}, 6 \mathrm{H}) \mathrm{ppm} ;{ }^{13} \mathrm{C}$ NMR $(101$ $\left.\mathrm{MHz}, \mathrm{CDCl}_{3}\right) \delta=194.61,154.71,143.83,139.63,138.35,129.33,128.83,128.44$ $128.32,128.01,127.24,127.10,126.37,126.26,104.65,81.00,57.88,50.31,42.25$, 41.83, 33.20, 29.19, 27.77 ppm; MS (ESI) $m / z 423\left(M+\mathrm{H}^{+}, 100\right)$; Anal. Calcd for $\mathrm{C}_{29} \mathrm{H}_{30} \mathrm{~N}_{2} \mathrm{O}: \mathrm{C}, 82.43 ; \mathrm{H}, 7.16$; N, 6.63; Found: C, 82.48; H, 6.98; N, 6.45.

\section{1,3-dibenzyl-1,2,3,4,7,8-hexahydro-7,7-dimethyl-2-phenylquinazolin-5(6H)-one}

(6\{5,4,1\}) 67\% yield, white solid, Mp: 157-158 ${ }^{\circ} \mathrm{C}$; IR $v_{\max }(\mathrm{KBr}): 2955,2360,2341$, $1652,1616,1587,1558,1505,1495,1471,1436,1395,1361,1297,1173,1121,1028$, 
937, 892, 740, 698, $669 \mathrm{~cm}^{-1} ;{ }^{1} \mathrm{H}$ NMR (400 MHz, $\left.\mathrm{CDCl}_{3}\right) \delta=7.52-7.06(\mathrm{~m}, 15 \mathrm{H})$, $4.86(\mathrm{~d}, J=16.7 \mathrm{~Hz}, 2 \mathrm{H}), 3.99(\mathrm{~d}, J=16.7 \mathrm{~Hz}, 1 \mathrm{H}), 3.74(\mathrm{~d}, J=13.5 \mathrm{~Hz}, 1 \mathrm{H}), 3.63$ (m, 2H), $3.25(\mathrm{~d}, J=16.4 \mathrm{~Hz}, 1 \mathrm{H}), 2.69(\mathrm{~d}, J=16.2 \mathrm{~Hz}, 1 \mathrm{H}), 2.58(\mathrm{~d}, J=16.2 \mathrm{~Hz}$ $1 \mathrm{H}), 2.35(\mathrm{~d}, J=16.3 \mathrm{~Hz}, 1 \mathrm{H}), 2.28(\mathrm{~d}, J=16.3 \mathrm{~Hz}, 1 \mathrm{H}), 1.23(\mathrm{~d}, J=23.3 \mathrm{~Hz}, 6 \mathrm{H})$ ppm; ${ }^{13} \mathrm{C}$ NMR $\left(101 \mathrm{MHz}, \mathrm{CDCl}_{3}\right) \delta=193.11,156.33,138.51,138.47,137.13$, $128.92,128.71,128.53,128.18,128.16,127.90,127.04,127.00,126.87,102.04$, 58.04, 50.89, 49.54, 41.47, 39.68, 32.59, 30.00, 27.56 ppm; MS (ESI) $m / z 437(M+$ $\mathrm{H}^{+}, 100$ ); Anal. Calcd for $\mathrm{C}_{30} \mathrm{H}_{32} \mathrm{~N}_{2} \mathrm{O}$ : C, 82.53; H, 7.39; N, 6.42; Found: C, 82.86; $\mathrm{H}$, $7.38 ; \mathrm{N}, 6.10$.

\section{${ }^{1} \mathrm{H}$ and ${ }^{13} \mathrm{C}$ spectra of octahydroquinazoline-5-ones 6 in Table 2}

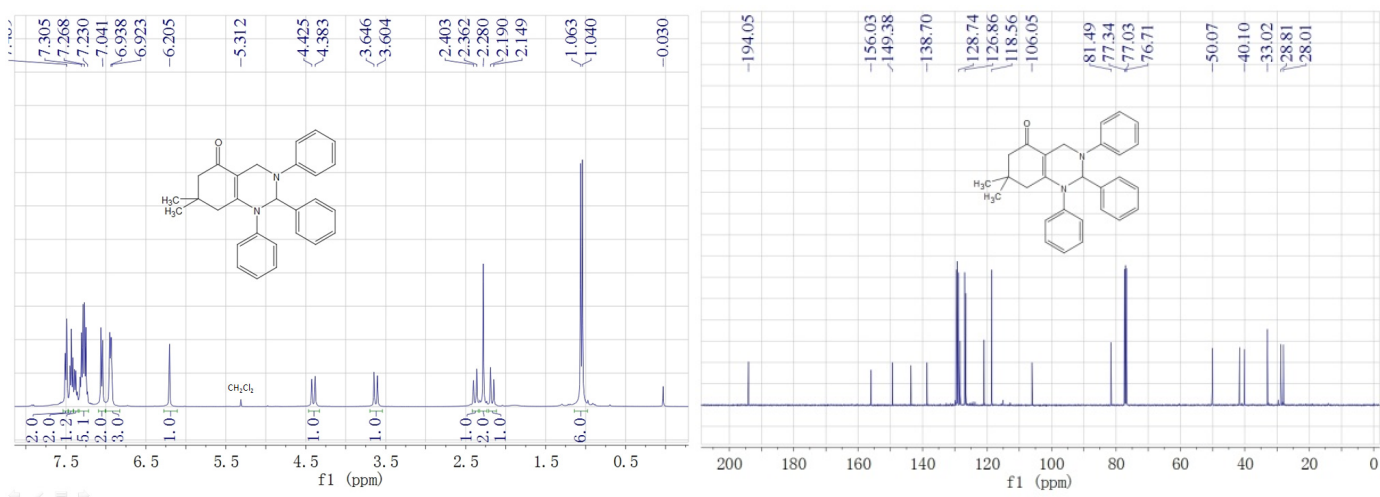

(1) ${ }^{1} \mathrm{H}$ NMR and ${ }^{13} \mathrm{C}$ NMR spectra of $6\{1,1,1\}$

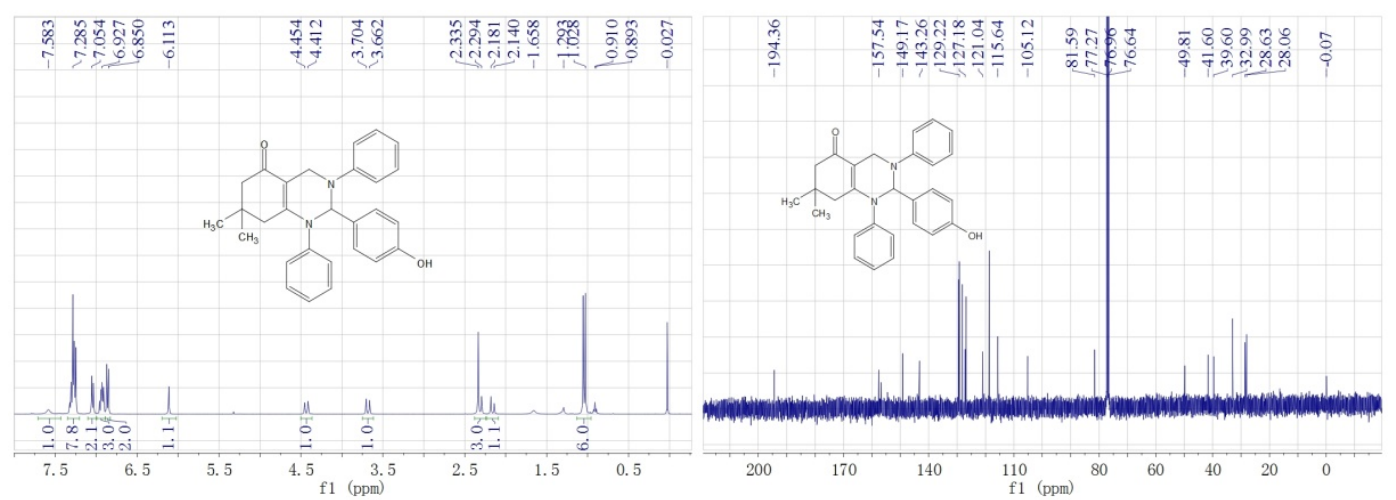

(2) ${ }^{1} \mathrm{H}$ NMR and ${ }^{13} \mathrm{C}$ NMR spectra of $6\{1,1,2\}$ 


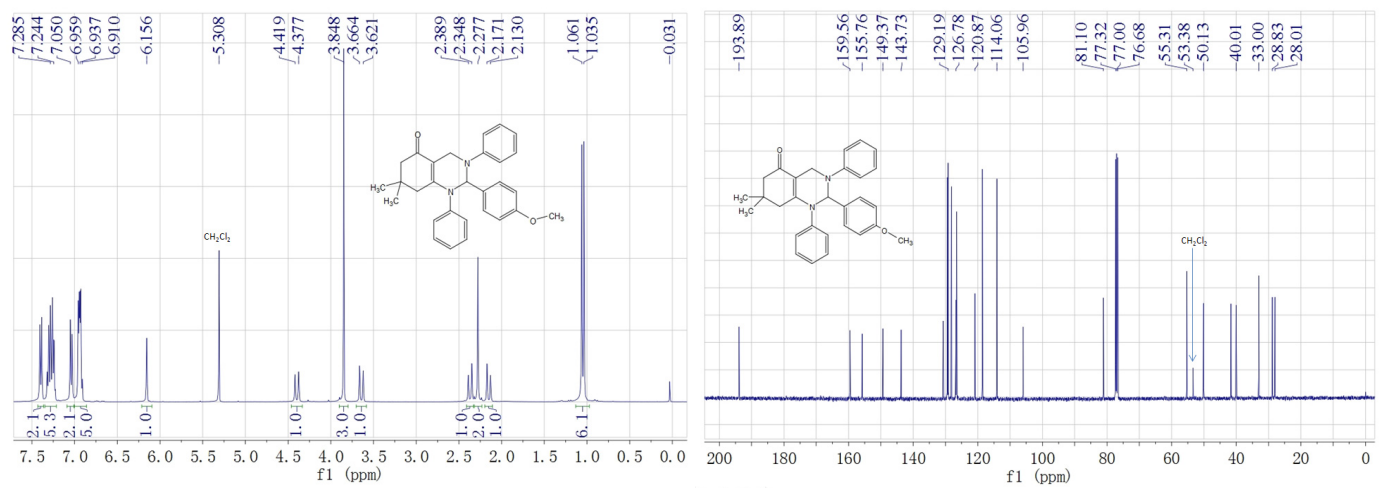

(3) ${ }^{1} \mathrm{H}$ NMR and ${ }^{13} \mathrm{C}$ NMR spectra of $6\{1,1,3\}$

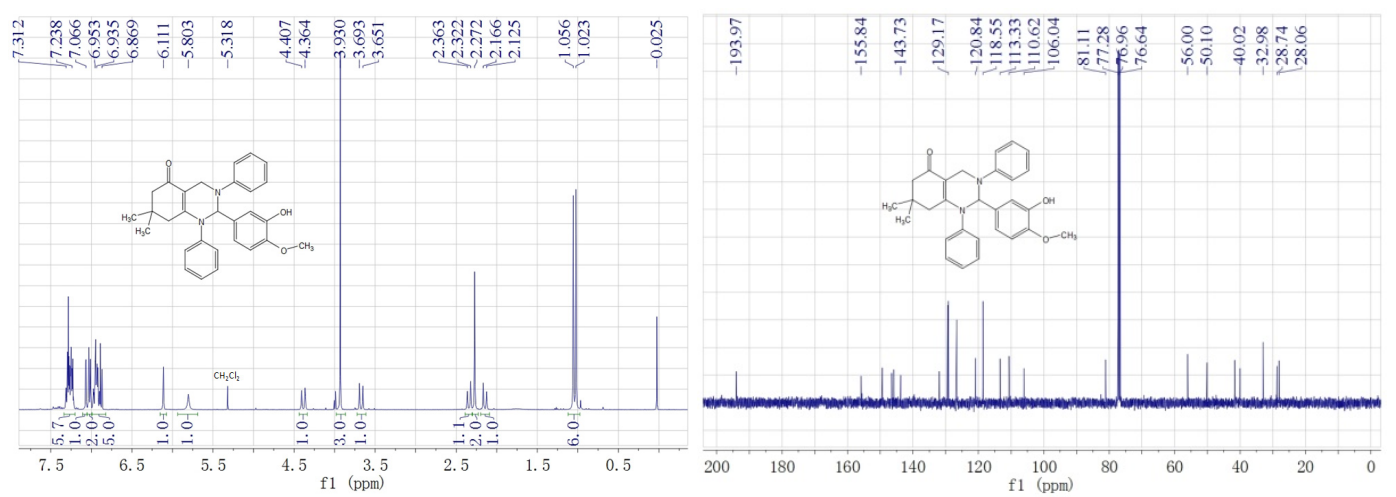

(4) ${ }^{1} \mathrm{H}$ NMR and ${ }^{13} \mathrm{C}$ NMR spectra of $6\{1,1,4\}$

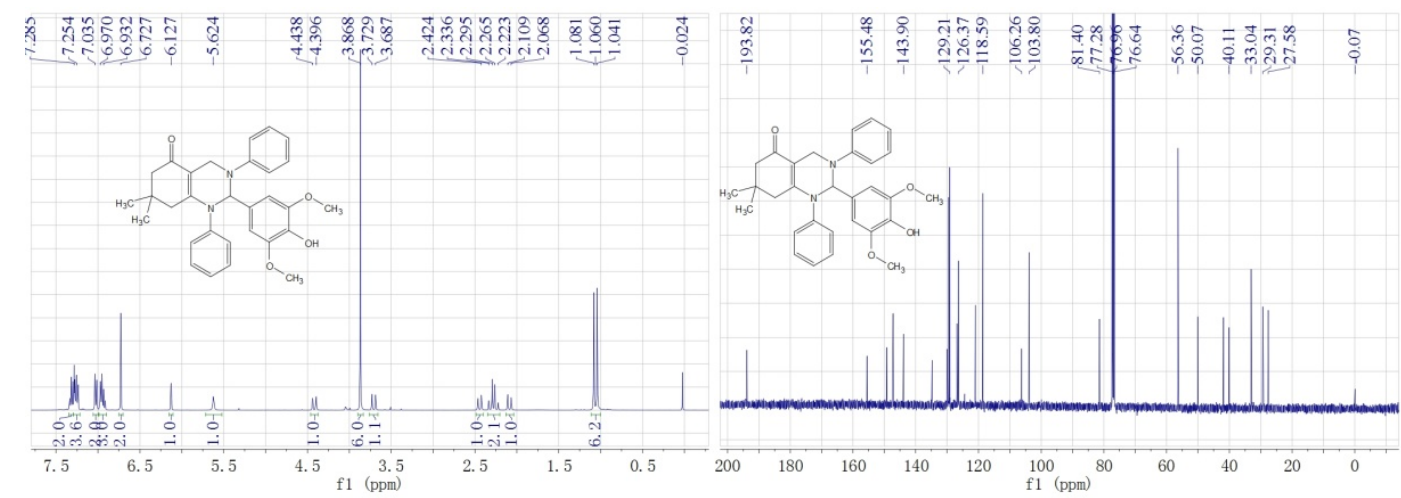

(5) ${ }^{1}$ H NMR and ${ }^{13} \mathrm{C}$ NMR spectra of $6\{1,1,5\}$

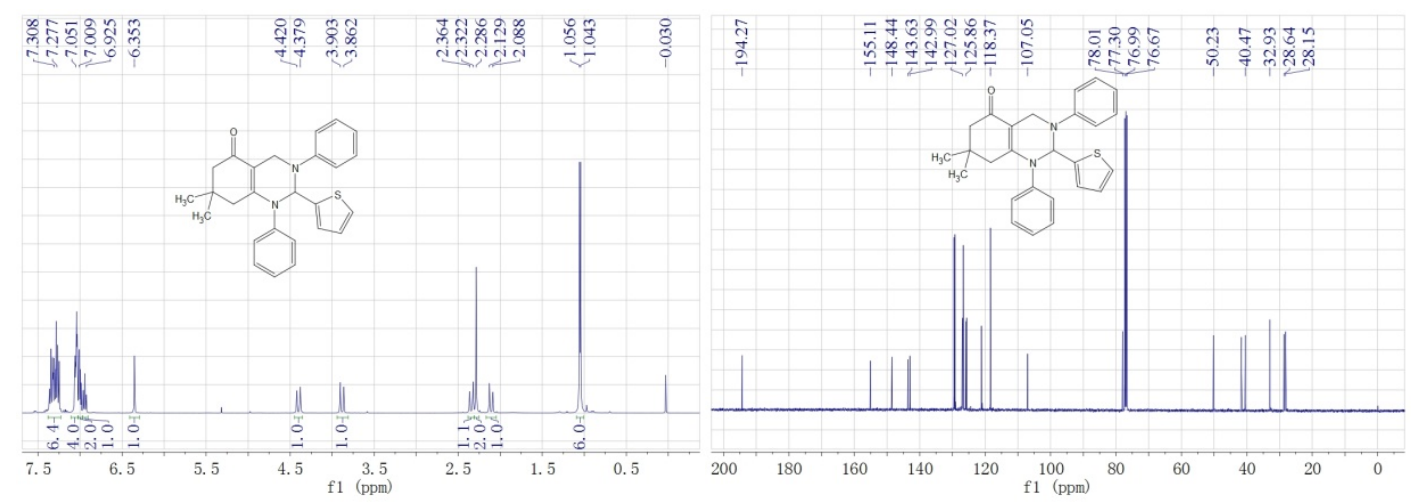

(6) ${ }^{1} \mathrm{H}$ NMR and ${ }^{13} \mathrm{C}$ NMR spectra of $6\{1,1,6\}$ 


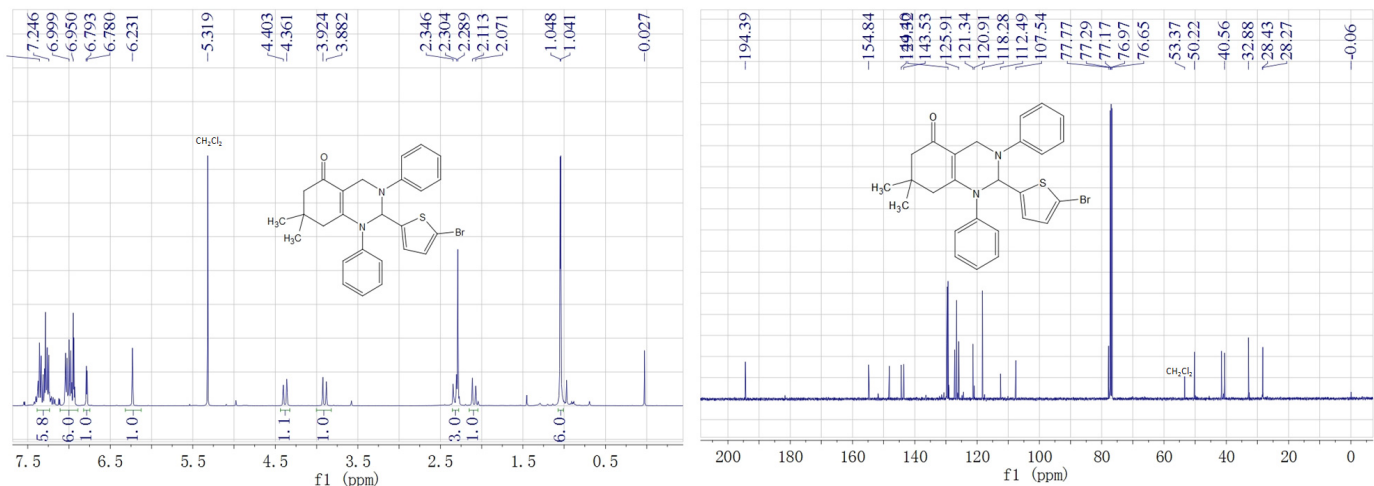

(7) ${ }^{1} \mathrm{H}$ NMR and ${ }^{13} \mathrm{C}$ NMR spectra of $6\{1,1,7\}$

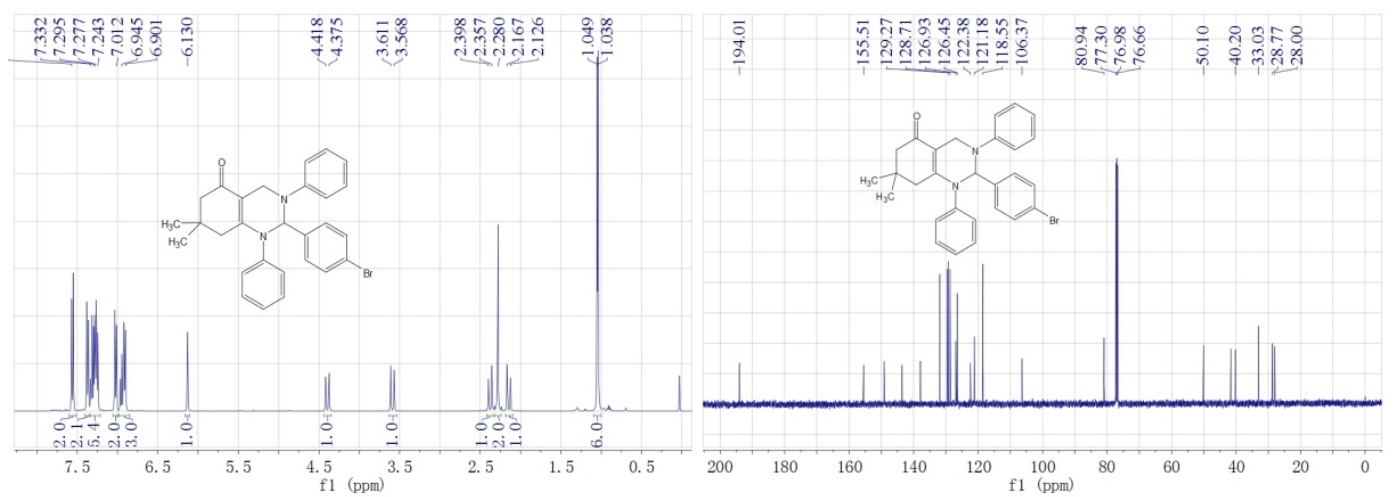

(8) ${ }^{1} \mathrm{H}$ NMR and ${ }^{13} \mathrm{C}$ NMR spectra of $6\{1,1,8\}$

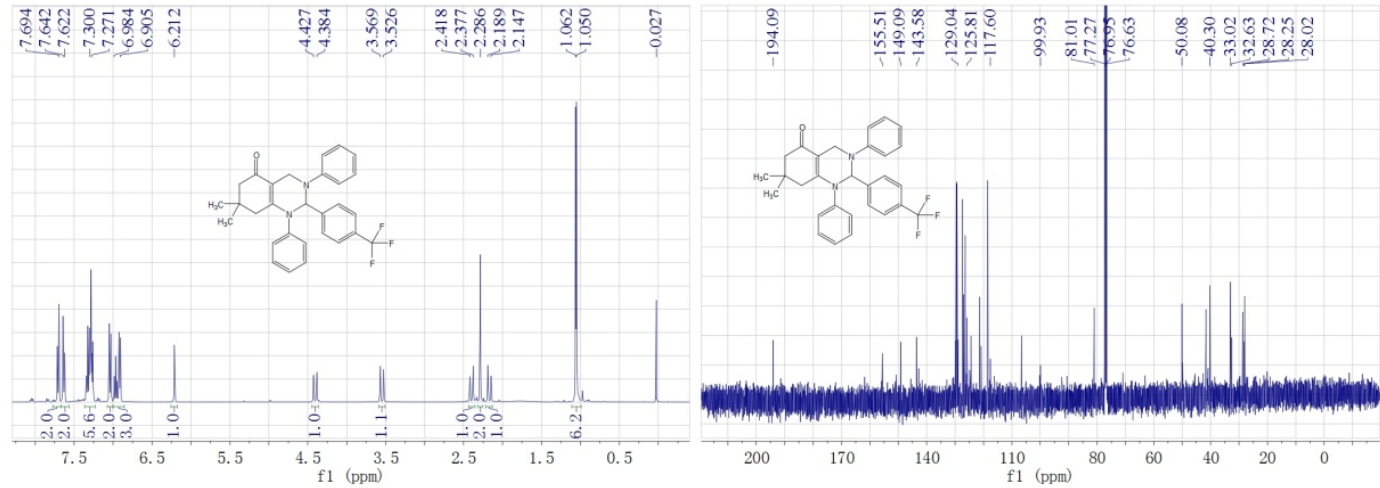

(9) ${ }^{1} \mathrm{H}$ NMR and ${ }^{13} \mathrm{C}$ NMR spectra of $6\{1,1,9\}$

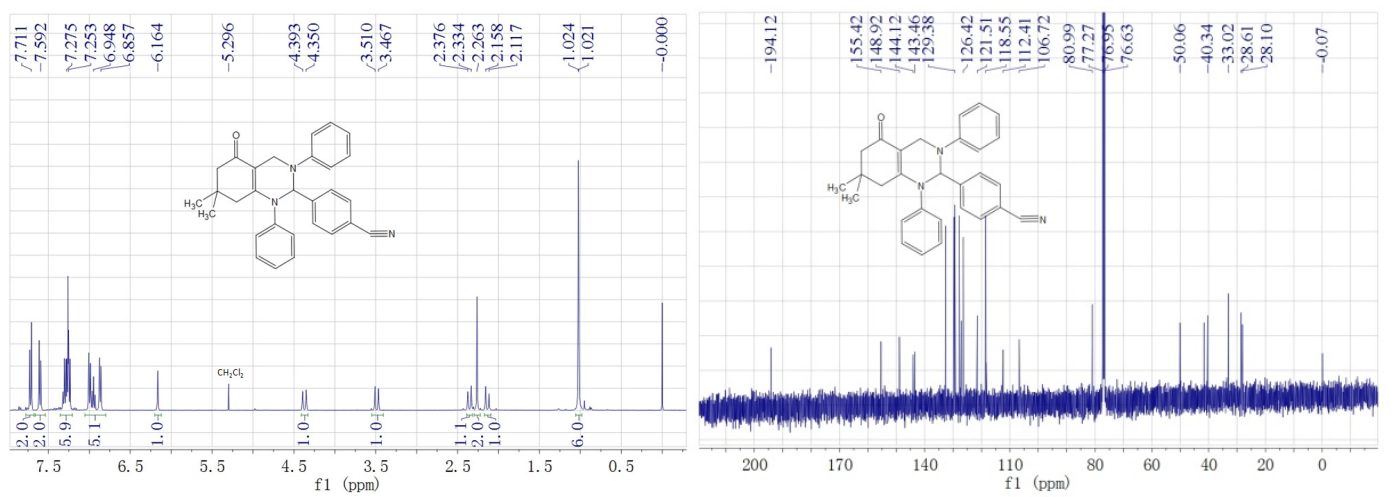

(10) ${ }^{1} \mathrm{H}$ NMR and ${ }^{13} \mathrm{C}$ NMR spectra of $6\{1,1,10\}$ 


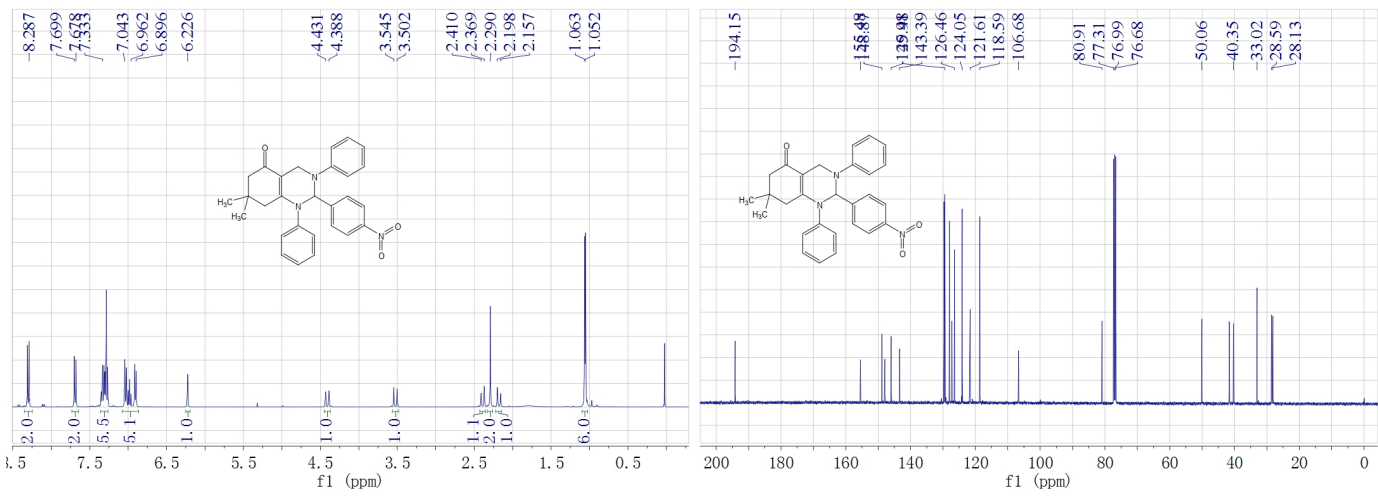

(11) ${ }^{1} \mathrm{H}$ NMR and ${ }^{13} \mathrm{C}$ NMR spectra of $6\{1,1,11\}$

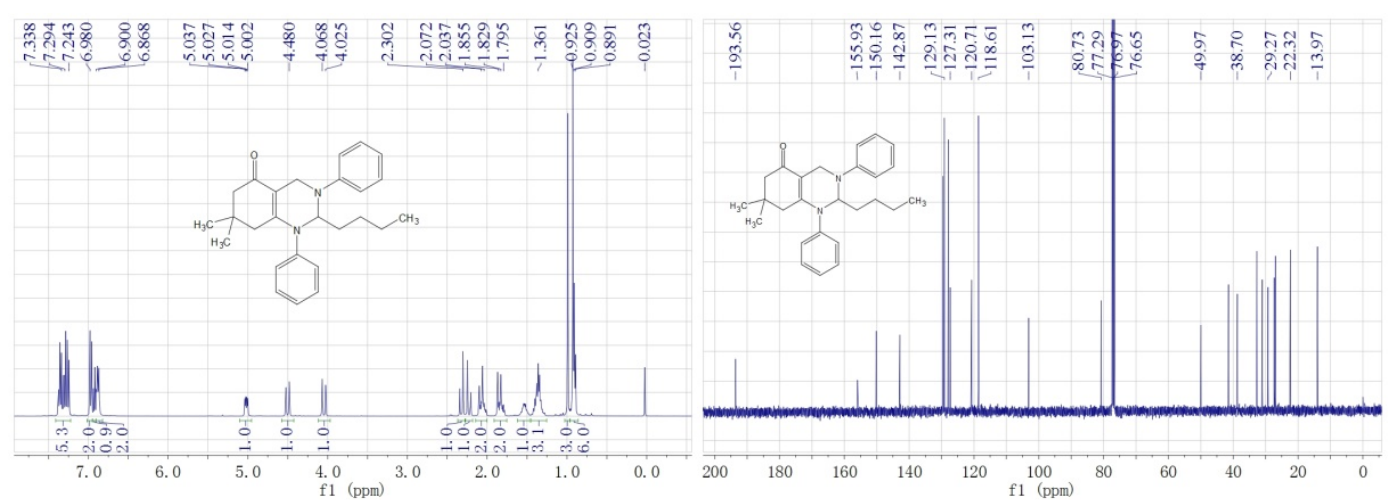

(12) ${ }^{1} \mathrm{H}$ NMR and ${ }^{13} \mathrm{C}$ NMR spectra of $6\{1,1,12\}$

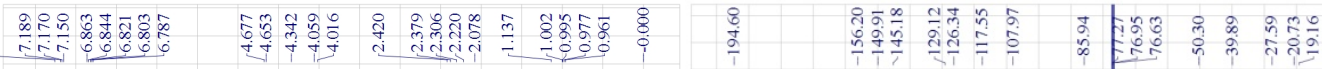

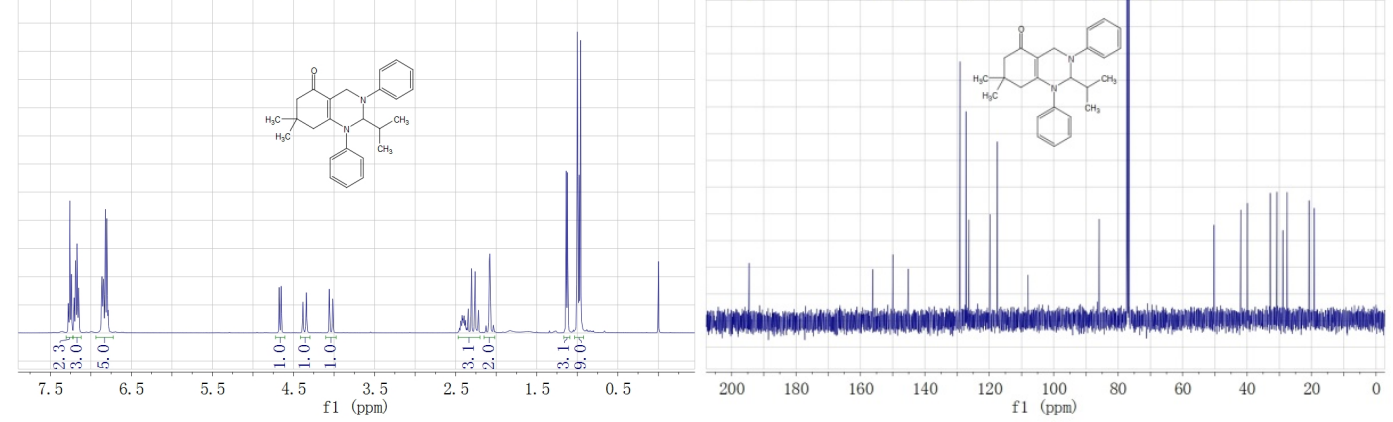

(13) ${ }^{1} \mathrm{H}$ NMR and ${ }^{13} \mathrm{C}$ NMR spectra of $6\{1,1,13\}$

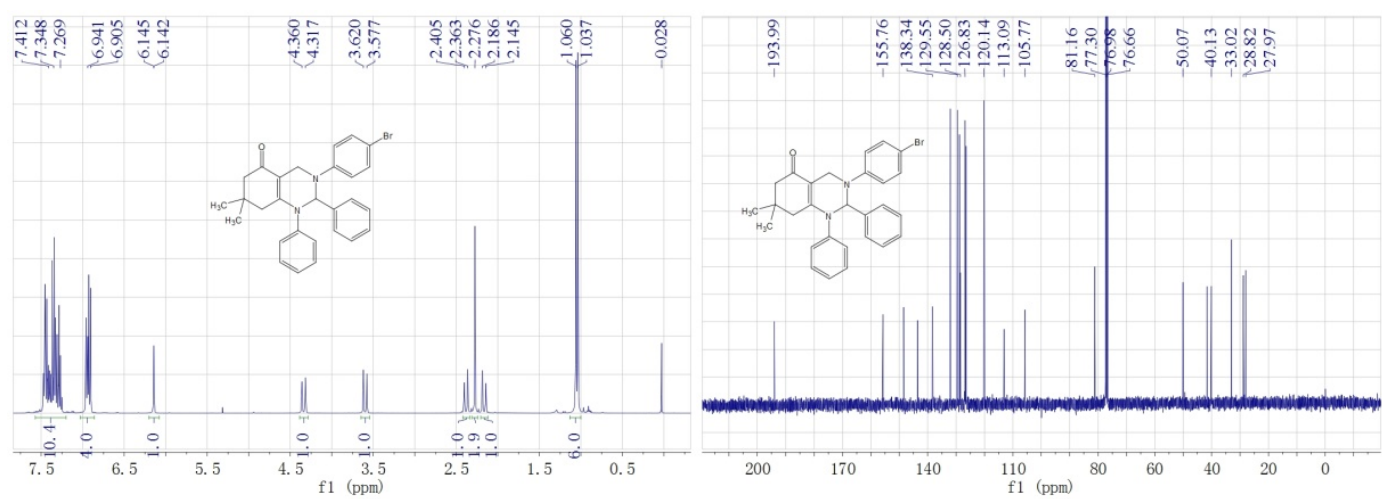

(14) ${ }^{1} \mathrm{H}$ NMR and ${ }^{13} \mathrm{C}$ NMR spectra of $6\{1,2,1\}$ 


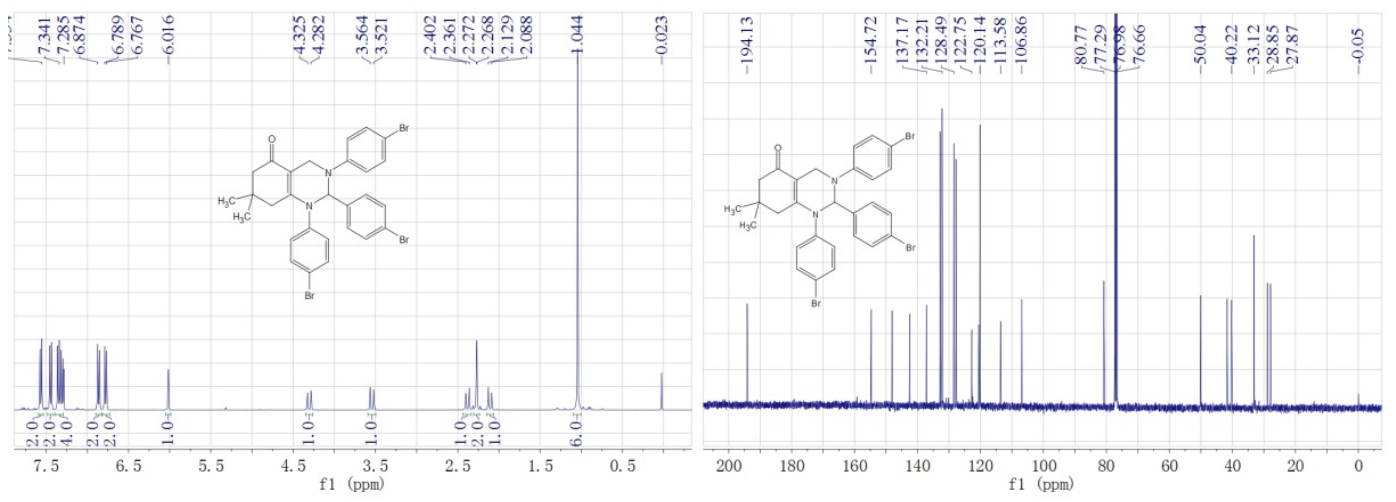

(15) ${ }^{1} \mathrm{H}$ NMR and ${ }^{13} \mathrm{C}$ NMR spectra of $6\{2,2,8\}$

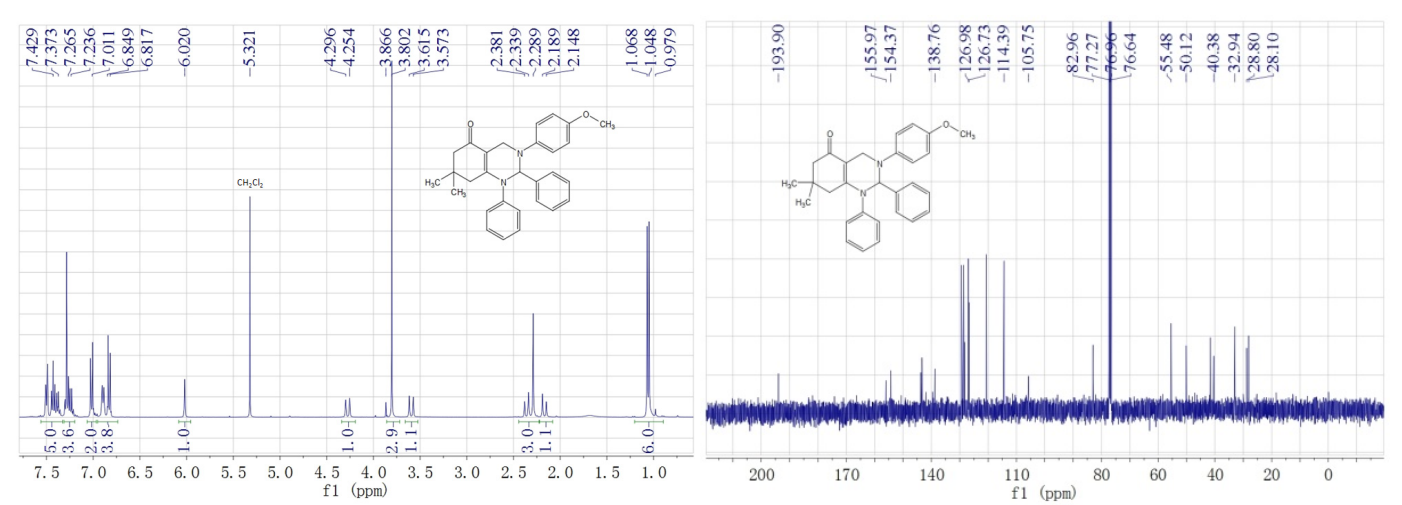

(16) ${ }^{1} \mathrm{H}$ NMR and ${ }^{13} \mathrm{C}$ NMR spectra of $6\{1,3,1\}$

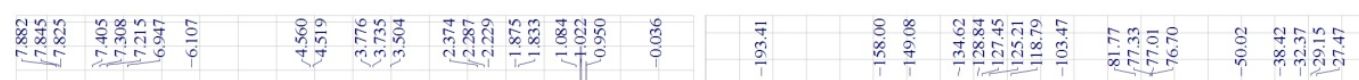

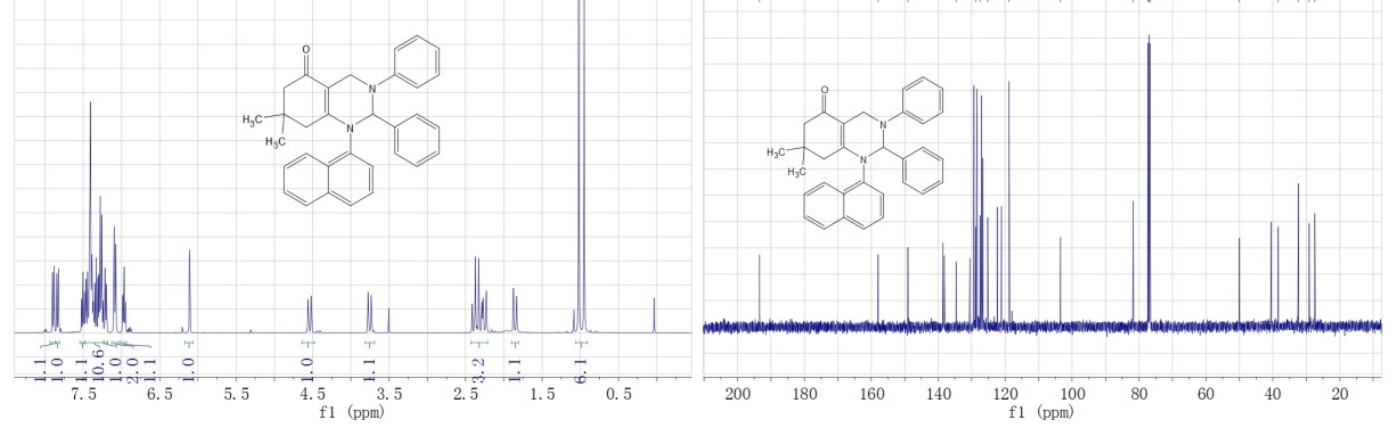

(17) ${ }^{1} \mathrm{H}$ NMR and ${ }^{13} \mathrm{C}$ NMR spectra of $6\{3,1,1\}$

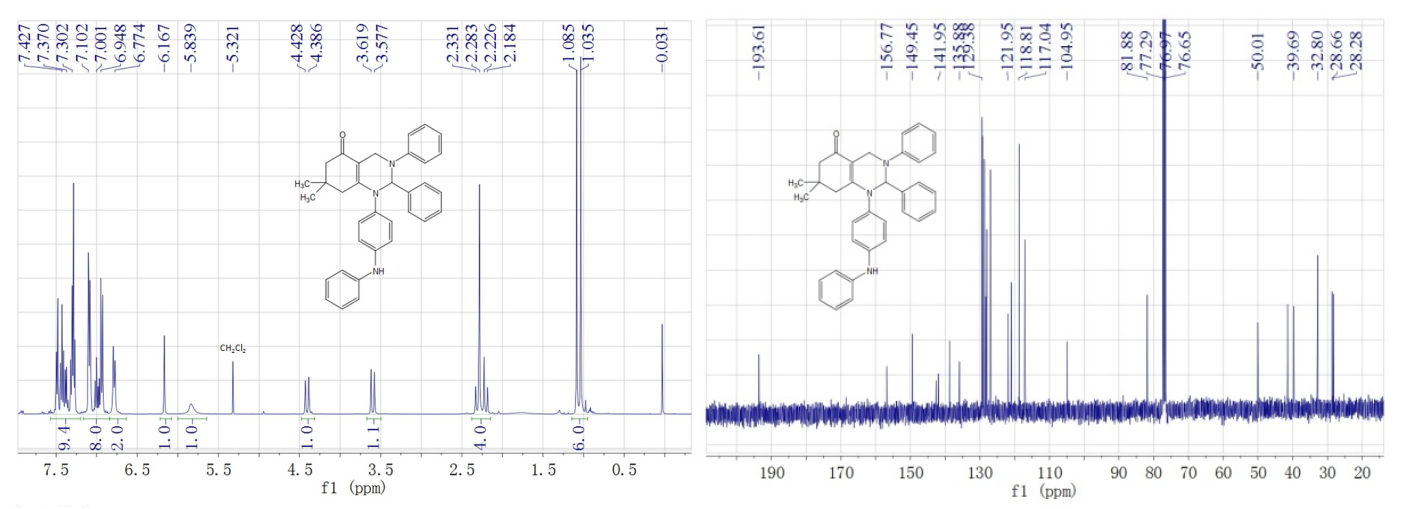

(18) ${ }^{1} \mathrm{H}$ NMR and ${ }^{13} \mathrm{C}$ NMR spectra of $6\{4,1,1\}$ 


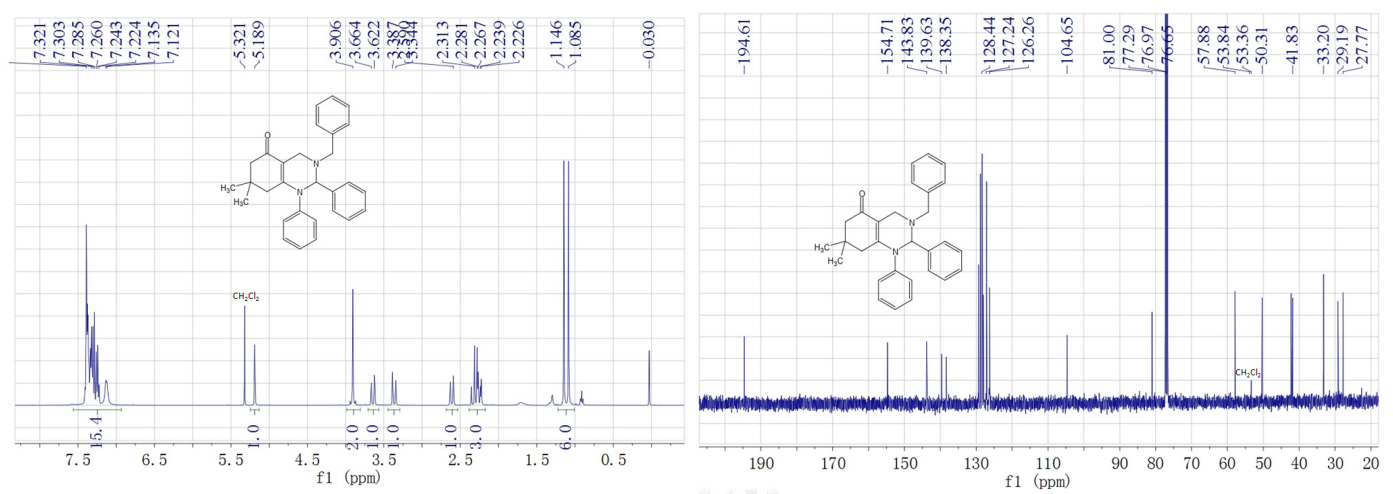

(19) ${ }^{1} \mathrm{H}$ NMR and ${ }^{13} \mathrm{C}$ NMR spectra of $6\{1,4,1\}$

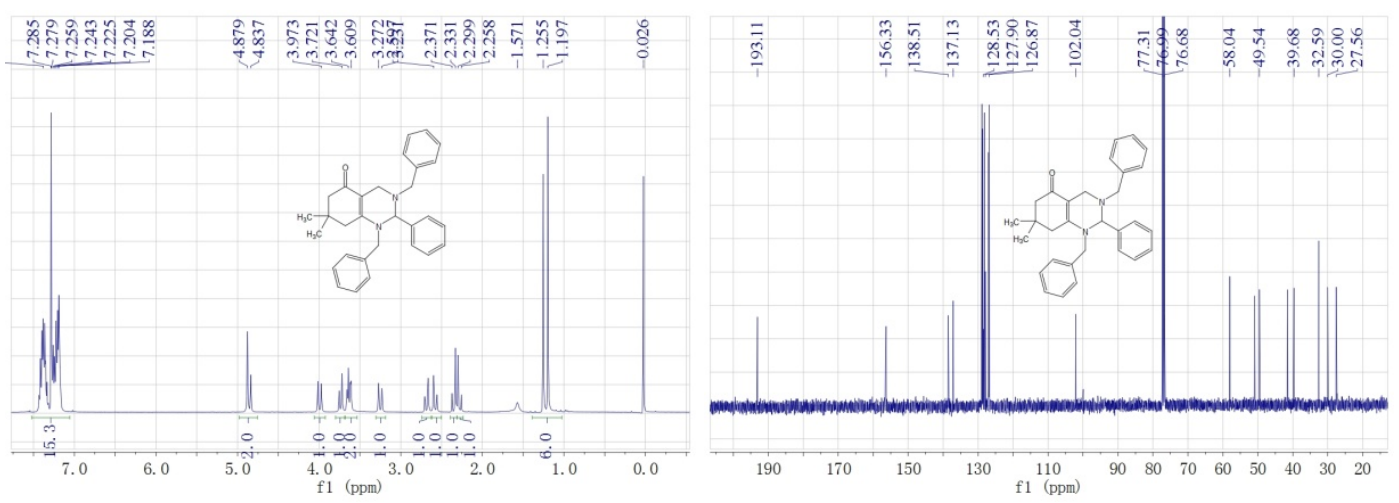

(20) ${ }^{1} \mathrm{H}$ NMR and ${ }^{13} \mathrm{C}$ NMR spectra of $6\{5,4,1\}$ 